\title{
Early clinical predictors of pneumonia in critically ill spinal cord injured individuals: a retrospective cohort study
}

\author{
Jacqui Agostinello ${ }^{1}$ Camila R. Battistuzzo ${ }^{1} \cdot$ Peter E. Batchelor ${ }^{1}$
}

Received: 17 April 2018 / Revised: 18 August 2018 / Accepted: 29 August 2018 / Published online: 27 September 2018

(c) International Spinal Cord Society 2018

\begin{abstract}
Study design Retrospective cohort.

Objectives Pneumonia is the dominant complication following traumatic spinal cord injury (SCI) and profoundly impacts morbidity by prolonging length of stay and worsening neurological outcome. The aims of this study were to determine the key predictors of clinically important pneumonia (CIP); and to examine the impact of CIP on resource utilisation in critically ill acute traumatic SCI individuals between 2010 and 2015.

Setting Alfred and Austin Hospitals (Melbourne, Australia).

Methods Data were extracted from the medical records of 93 cases of acute traumatic SCI resulting in ISNCSCI C3-L1 level of injury requiring admission to the intensive care unit and aged between 15 and 70 years. Patients with life-threatening injuries, not requiring spinal surgery, palliated within 7 days of injury, diagnosis of traumatic central cord syndrome or with poor general health, were excluded.

Results A total of 33 episodes of CIP were observed. Median time to CIP diagnosis was $65 \mathrm{~h}$ (IQR: 42-93) and median time to spinal surgery was $22 \mathrm{~h}$ (IQR: 12-32). Four key predictors were identified; male gender (OR: 18.3, CI: 1.9-174.9, $p=$ 0.001 ), motor complete injury (OR: 10.1 , CI: $1.1-92.1, p=0.011$ ), presence of chest trauma (OR: 4.5 , CI: 1.4-14.4, $p=$ 0.007 ) and delayed intubation (HR: 6.8, CI: 1.6-28.6, $p=0.009$ ).

Conclusions This study identifies four key predictors involved in elevated pneumonia risk; male gender, motor complete injury, presence of chest trauma and delayed intubation, enabling the future synthesis of a pneumonia prediction tool for use in the acute postinjury period.
\end{abstract}

\section{Introduction}

Respiratory insufficiency is the leading cause of morbidity and mortality following acute traumatic spinal cord injury (SCI) [1], peaking during the first 5 days following trauma as a result of severe muscle weakness, chest injuries, spinal and neurogenic shock [2]. Pneumonia is highly prevalent in the acute hospitalisation phase following SCI [3], however, reported incidence rates are dependent on diagnostic criteria

Electronic supplementary material The online version of this article (https://doi.org/10.1038/s41393-018-0196-6) contains supplementary material, which is available to authorised users.

Jacqui Agostinello

j.agostinello@alfred.org.au

1 Department of Medicine (Royal Melbourne Hospital), The University of Melbourne, Royal Parade, VIC, Australia and have a large range (11-84\%) [4, 5]. SCI individuals who develop pneumonia have been shown to have a longer acute hospital length of stay (LOS) [6], therefore early awareness of risk may assist with limiting severity or presence of infection, improving resource allocation and reducing overall hospital costs.

Moderate and severe pneumonia resulting in a sustained elevation of temperature has the most potential to cause severe hypoxia and secondary SCI, impacting neurological outcome [7]. However, no studies to date have separated moderate and severe pneumonia from mild infections and identified key predictors. As SCI individuals are particularly susceptible to respiratory tract infections, results from studies including able-bodied participants are difficult to extrapolate. Although the strongest predictor of respiratory complications in SCI individuals is surgical timing [8], regular volume restoration physiotherapy treatment (VRPT) [3], injury severity, comorbidity burden, advanced age [9] and alcohol intake [10] have also been shown to play a role. 
Current surgical timing in SCI has been established $[11,12]$, however, other interventions hypothesised to influence pneumonia risk such as the timing of intubation, mobilisation and VRPT lack standardisation and are poorly defined in the literature. Without established timeframes for common interventions, clinical translatability is challenging.

The aims of this study were to determine the key predictors of clinically important pneumonia (CIP); and to examine the impact of CIP on resource utilisation in critically ill acute traumatic SCI individuals admitted between 2010 and 2015.

\section{Methods}

\section{Study design}

A retrospective data audit of SCI cases admitted between July 2010 and December 2015 to the Alfred and Austin Hospitals (Melbourne, Victoria, Australia) was conducted. Low-risk Human Research Ethical Approval was obtained at both institutions.

\section{Inclusion and exclusion criteria}

Patients were included in the study if they had an acute traumatic SCI resulting in C3-L1 neurological level of injury according to the International Standards for the Neurological Classification of SCI (American Spinal Injury Association Impairment Scale (AIS) A-D), required admission to the intensive care unit (ICU) and were aged $15-70$ years.

Patients were excluded if they did not require spinal surgery, had poor general health (e.g., pre-existing neurological deficits, past history of ankylosing spondylitis), when key information was missing (e.g., time of injury and surgery) or when palliation occurred within the first 7 days. Patients with traumatic central cord syndrome (TCCS) were also excluded to minimise bias related to the differences in management and surgical decision making in SCI individuals with a stable spinal column. TCCS was defined as evidence of cervical spinal cord trauma on magnetic resonance imaging with cord compression resulting from spondylosis or congenital stenosis (not fracture or fracture dislocation), with or without acute disco-ligamentous injury narrowing the cervical canal.

Patients sustaining life-threatening injuries (those that confounded initial International Standards for the Neurological Classification of Spinal Cord Injury (ISNCSCI) assessment or delayed surgical intervention), defined as significant head injury at the scene (sustained Glasgow Coma Score $<13$ ), significant chest trauma (persistent severe hypoxia or haemodynamic instability following chest tube insertion), haemodynamic instability resulting from abdominal or retro-peritoneal bleeding, pelvic fracture or more than two long bone fractures were also excluded.

Patients with non-life-threatening injuries were termed polytrauma (e.g., rib or sternal fractures, stable organ injuries and peripheral limb fractures) and remained included if they otherwise fulfilled the inclusion and exclusion criteria.

\section{Data collection}

A senior physiotherapist collected the following baseline data fields for each included case: age, gender, ISNCSCI neurological level and AIS grade at acute hospital admission, smoking history, other injuries, Charlston comorbidity index (CCI) score, weight, first $\mathrm{pH}$, direct or indirect surgical hospital admission, percentage of time spent supine and frequency of position changes per day for the first 14 days. Time-dependent data fields were also collected: time to spinal surgery, first turn, intubation, first VRPT, optimal VRPT, first physiotherapy assessment and to spinal clearance (definitions of all included predictors are described in Table 1).

Resource utilisation data fields were collected: acute hospital LOS (date and time of acute hospital admission and discharge), ICU LOS (date and time of ICU admission and discharge), duration of ventilation (date and time of intubation and successful extubation) and time to first mobilisation (first sit out of bed or on the edge of bed). Additionally, number of bronchoscopies and number of chest X-rays were collected during the first 14 days following injury and further descriptive data were sought to assist with definition of current intubation practices (intubation reason and location).

Additionally, date and time of CIP onset (defined under data analysis), date and time of resolution (end of antibiotic therapy, or in the absence of antibiotic therapy end of clinical symptoms) and maximum temperature rise were collected.

\section{Data analysis}

Data were separated into two main categories of interest: baseline demographics and injury characteristics (present on acute hospital admission) and clinical features of the individual's care following acute hospital admission.

CIP was defined as clinical, radiological or microbiological diagnosis of pneumonia based on Centers for Disease Control [13] with $\mathrm{PaO}_{2} / \mathrm{FiO}_{2} 150-240$ for $\geq 12 \mathrm{~h}$ or $\mathrm{PaO}_{2} / \mathrm{FiO}_{2}<150$, and/or core or tympanic temperature $\geq 38.5{ }^{\circ} \mathrm{C}$ (where temperature control not present) and/or induction of significant haemodynamic disturbance requiring inotropic support or resulting in death. CIP onset was 
Table 1 Operational definitions of predictors

\begin{tabular}{|c|c|c|c|}
\hline $\begin{array}{l}\text { Statistical analysis } \\
\text { category }\end{array}$ & $\begin{array}{l}\text { Explanatory } \\
\text { variable }\end{array}$ & Definition & Coding \\
\hline \multirow{16}{*}{$\begin{array}{l}\text { Baseline } \\
\text { characteristics }\end{array}$} & Age & Age in decades & Years \\
\hline & \multirow[t]{2}{*}{ Gender } & Male & 0 \\
\hline & & Female & 1 \\
\hline & \multirow{4}{*}{$\begin{array}{l}\text { ISNCSCI level of } \\
\text { injury }\end{array}$} & Upper cervical (C3-4) & 0 \\
\hline & & Lower cervical (C5-8) & 1 \\
\hline & & Upper thoracic (T1-8) & 2 \\
\hline & & Thoracolumbar (T9-L1) & 3 \\
\hline & \multirow[t]{2}{*}{ Admission AIS } & Motor complete (AIS A or B) & 1 \\
\hline & & Motor incomplete (AIS C or D) & 0 \\
\hline & \multirow[t]{2}{*}{ Smoking history } & History of smoking (past or present) & 1 \\
\hline & & No history of smoking & 0 \\
\hline & \multirow{2}{*}{$\begin{array}{l}\text { Presence of } \\
\text { polytrauma }\end{array}$} & Presence of other non-life-threatening injuries & 1 \\
\hline & & Isolated SCI & 0 \\
\hline & \multirow{2}{*}{$\begin{array}{l}\text { Presence of chest } \\
\text { trauma }\end{array}$} & Presence of pneumothorax and/or haemothorax & 1 \\
\hline & & Absence of pneumothorax and/or haemothorax & 0 \\
\hline & $\begin{array}{l}\text { Comorbidity } \\
\text { burden }\end{array}$ & Charlston comorbidity index score & $\begin{array}{l}\text { Units } \\
(0-39)\end{array}$ \\
\hline \multirow[t]{5}{*}{$\begin{array}{l}\text { Time-dependent } \\
\text { characteristics }\end{array}$} & $\begin{array}{l}\text { Time to spinal } \\
\text { surgery }\end{array}$ & $\begin{array}{l}\text { Time of injury to the midpoint between initiation } \\
\text { and completion of surgery }\end{array}$ & Hours \\
\hline & Time to first turn & $\begin{array}{l}\text { Time of injury to the first change in position to } \\
\text { side-lying (excluding log rolling for pressure area } \\
\text { care) }\end{array}$ & Hours \\
\hline & Time to intubation & $\begin{array}{l}\text { Time of injury to the time of intubation. If patient } \\
\text { was intubated solely for the duration of surgery, } \\
\text { an intubation time was not collected }\end{array}$ & Hours \\
\hline & Time to first VRPT & Time of injury to first VRPT & Hours \\
\hline & $\begin{array}{l}\text { Time to optimal } \\
\text { VRPT }\end{array}$ & $\begin{array}{l}\text { Time of injury to fifth VRPT in cervical injury, } \\
\text { time of injury to third VRPT in thoracolumbar } \\
\text { injury }\end{array}$ & Hours \\
\hline
\end{tabular}

ISNCSCI International Standards for the Neurological Classification of Spinal Cord Injury, AIS American Spinal Injury Association Impairment Scale, SCI spinal cord injury, VRPT volume restoration physiotherapy treatment defined as the date and time of severe acute desaturation. In the absence of severe acute desaturation, the date and time of first fever utilised by medical staff for diagnosis was used. If the individual experienced more than one episode of CIP, the time of the first episode was used.

Hospital-acquired pneumonia (HAP) was defined as acute hospital admission to pneumonia onset being $\geq 48 \mathrm{~h}$. VRPT was defined as the application of positive pressure to enable an increased tidal volume, and encompassed techniques such as ventilator or manual hyperinflation, intermittent positive pressure breathing, in/exsufflation and noninvasive ventilation.

Transfer to the ICU was generally instigated to enable close monitoring of respiratory and cardiovascular function in the setting of respiratory and/or cardiovascular instability. Due to the risk of diaphragmatic mechanical disadvantage in sitting, acute general rehabilitation was not performed as a priority in our cohort unless their ISNCSCI was below T8 or their respiratory status was stable, e.g., no CIP. Examining standard physiotherapy practice (e.g., mobilisation versus respiratory techniques) in greater detail was difficult due to internal bias in clinical decision making.

\section{Statistical analysis}

Potential predictors were first identified from a review of the literature and then limited to those that were consistently documented in the individual's medical record. As part of this process, weight, first $\mathrm{pH}$, time to spinal clearance, time to first physiotherapy assessment, percentage of time spent supine and frequency of position changes for the first 14 days were excluded. Due to poor documentation of weight in the acute setting, we were unable to incorporate analysis of the impact of specific weaning strategies such as high tidal volume ventilation on CIP risk. Please see Table 1 for operational definitions of all included predictors. 
Eight baseline characteristics (age in decades, gender, admission ISNCSCI level of injury, admission AIS grade, smoking history, presence of polytrauma, presence of chest trauma and comorbidity burden) were entered into an all subset regression model to identify key predictors using GenStat version 16 software. The "best" model with the smallest Akaike information criterion (AIC) was identified and the first parsimonious model was identified by considering simpler models with an AIC within two points of the "best" model [14]. The key predictors in the most parsimonious model were then used in a final multivariate logistic regression model.

Five time-dependent explanatory variables (time to surgery, first turn, intubation, first VRPT and optimal VRPT) were then individually introduced into a univariate Cox regression survival analysis modelling time to CIP diagnosis using IBM SPSS version 22 software. Time to initiation of surgery and time to intubation were also introduced together in a multivariate Cox regression to ascertain how these two variables interacted.

A $p$-value of $<0.05$ was considered statistically significant. Odds ratios (ORs), hazard ratios (HRs) and their 95\% confidence intervals (CIs) were then calculated for each predictor.

Baseline clinical characteristics and resource utilisation data (no CIP versus CIP) were compared using Mann-Whitney $U t$-test and chi-square test with Prism software (version 6, GraphPad, CA, USA). Significance was set at $p<0.05$. Data are presented as median \pm interquartile range (IQR).

\section{Results}

\section{Screening of patients}

A total of 175 cases were screened and 93 cases were included. The reasons for exclusion were: life-threatening injuries $(n=38)$, key information (time of injury or time of surgery) unavailable $(n=14)$, TCCS $(n=21)$, no surgical intervention $(n=4)$, poor general health $(n=4)$ and palliation within the first 7 days $(n=1)$.

Some baseline differences were found between the small excluded group (key information unavailable) and the included sample (see Supplemental Information). This was a unique cohort because a significant proportion $(29 \%, n=$ 4) sustained their SCI internationally and required medical stabilisation and surgery outside of Australia. The rest of the sample $(n=10,71 \%)$ attended a non-involved hospital within Australia (majority of which were interstate) prior to being transferred to a SCI specialist centre.

\section{Demographics and clinical characteristics}

The median (IQR) age was 33 (22-53) years and the majority $(83 \%)$ of patients were male. The most frequent injury level was C5-8 (37\%) as a result of a motor vehicle accident $(27 \%)$ or fall from $\geq 1 \mathrm{~m}$ height $(23 \%)$. The majority of cases $(74 \%)$ were classified as motor complete (AIS A or B) SCI and a high proportion of individuals (72\%) had a pneumothorax or haemothorax on admission to hospital. Males had higher alcohol consumption than females overall, with 19 males $(25 \%, n=77)$ having significant alcohol intake immediately preceding injury versus 1 female $(16 \%, n=16)$. Predominant intubation reason was initiation of surgery $(n=49,53 \%)$. Most cases $(n=73,78 \%)$ required ongoing mechanical ventilation after the completion of surgery, and of these cases $(n=$ 73) a high proportion $(n=72,97 \%)$ did not require intubation until after acute hospital admission. There was a relatively equal distribution of patients admitted directly versus indirectly to a surgical hospital. Clinical characteristics of included patients are described in Table 2.

\section{Incidence of respiratory complications}

The most common respiratory complications were atelectasis $(n=71,76 \%)$ and pneumonia $(n=69,74 \%)$, with atelectasis tending to slightly precede pneumonia diagnosis. Thoracolumbar SCI cases had a higher incidence of pleural effusions, haemopneumothoraces, acute pulmonary oedema and pulmonary emboli compared with cervical SCI cases.

\section{Pneumonia characteristics}

A total of 33 episodes of CIP were observed during the study period, representing a $35 \%$ incidence rate. The overall median time to CIP diagnosis was $65 \mathrm{~h}$ (IQR: 42-93) and median duration of infection was 13 days (IQR: 8-19) (Fig. 1). Unsurprisingly, individuals who developed CIP had a significantly higher peak temperature rise compared with those who did not (median: $39.4{ }^{\circ} \mathrm{C}$ and IQR: 38.9-39.8 versus median: $38.7^{\circ} \mathrm{C}$ and IQR: 37.9-39.3, respectively, $p=<0.0001)$. All CIP cases survived to acute hospital discharge.

CIP was considered as hospital acquired in the majority of cases ( $n=23,70 \%$ ), and was generally diagnosed following $\geq 48 \mathrm{~h}$ of mechanical ventilation $(n=18,78 \%)$.

The overall median time to spinal surgery was $22 \mathrm{~h}$ (IQR: 12-32), and CIP cases underwent surgery at a median of $8 \mathrm{~h}$ later than non-CIP cases (20 h, IQR: 13-29 versus $28 \mathrm{~h}$, IQR: $12-41, p=0.0998)$, which trended towards significance. 
Table 2 Clinical characteristics of included patients

\begin{tabular}{|c|c|c|c|}
\hline Variable & $\begin{array}{l}\text { CIP cases, } \\
n=33 n(\%)\end{array}$ & $\begin{array}{l}\text { No CIP cases, } \\
n=60 n(\%)\end{array}$ & $p$-Value \\
\hline Age (median (IQR)) & $34(21-54)$ & $32(23-47)$ & 0.90 \\
\hline Male & $32(97)$ & $45(75)$ & 0.01 \\
\hline \multicolumn{4}{|l|}{ Accident category } \\
\hline Motor vehicle & $8(24)$ & $17(28)$ & 0.07 \\
\hline High fall ( $\geq 1 \mathrm{~m}$ ) & $6(18)$ & $15(25)$ & 0.45 \\
\hline Unprotected road users & $9(27)$ & $10(17)$ & 0.22 \\
\hline Water related & $6(18)$ & $6(10)$ & 0.26 \\
\hline $\begin{array}{l}\text { Struck by or collision with } \\
\text { object }\end{array}$ & $4(12)$ & $7(12)$ & 0.95 \\
\hline $\begin{array}{l}\text { Low fall (same level or }<1 \\
\text { m) }\end{array}$ & $0(0)$ & $5(8)$ & 0.09 \\
\hline \multicolumn{4}{|l|}{ ISNCSCI level of injury } \\
\hline C3-4 & $8(24)$ & $17(28)$ & 0.67 \\
\hline C5-8 & $14(42)$ & $20(33)$ & 0.38 \\
\hline T1-8 & $3(9)$ & $14(23)$ & 0.67 \\
\hline T9-L1 & $8(24)$ & $9(15)$ & 0.38 \\
\hline \multicolumn{4}{|l|}{ Acute admission AIS } \\
\hline Motor complete (A or B) & $32(97)$ & $46(77)$ & 0.01 \\
\hline Motor incomplete (C or D) & $1(3)$ & $14(42)$ & \\
\hline \multicolumn{4}{|l|}{ Presence of other injuries } \\
\hline Isolated & $10(30)$ & $29(48)$ & 0.09 \\
\hline Polytrauma & $23(70)$ & $31(53)$ & \\
\hline \multicolumn{4}{|l|}{ Presence of chest trauma } \\
\hline No & $20(61)$ & $47(78)$ & 0.07 \\
\hline Yes & $13(39)$ & $13(22)$ & \\
\hline \multicolumn{4}{|l|}{ Smoking history } \\
\hline No & $19(58)$ & $43(72)$ & 0.17 \\
\hline Yes & $14(42)$ & $17(28)$ & \\
\hline \multicolumn{4}{|l|}{$\mathrm{CCI}$} \\
\hline 0 & $17(52)$ & $35(58)$ & 0.53 \\
\hline 1 & $11(33)$ & $17(28)$ & 0.62 \\
\hline 2 & $4(12)$ & $4(7)$ & 0.40 \\
\hline 3 & $1(3)$ & $4(7)$ & 0.46 \\
\hline \multicolumn{4}{|l|}{ Surgical hospital admission } \\
\hline Indirect & $20(61)$ & $32(53)$ & 0.50 \\
\hline Direct & $13(39)$ & $28(47)$ & \\
\hline $\begin{array}{l}\text { Time to surgery (hours) } \\
\text { (median, (IQR)) }\end{array}$ & $28(12-41)$ & $20(13-29)$ & 0.10 \\
\hline $\begin{array}{l}\text { Time to first turn (hours) } \\
\text { (median, (IQR)) }\end{array}$ & $30(19-39)$ & $26(20-34)$ & 0.36 \\
\hline $\begin{array}{l}\text { Time to intubation (hours) } \\
\text { (median, (IQR)) }\end{array}$ & $7(4-23)$ & $9(5-18)$ & 0.55 \\
\hline $\begin{array}{l}\text { Time to first VRPT (hours) } \\
\text { (median, (IQR)) }\end{array}$ & $47(29-56)$ & $32(23-49)$ & 0.06 \\
\hline $\begin{array}{l}\text { Time to optimal VRPT } \\
\text { (hours) (median, (IQR)) }\end{array}$ & 75 (60-97) & $56(49-73)$ & 0.004 \\
\hline
\end{tabular}

$C I P$ clinically important pneumonia, IQR interquartile range, ISNCSCI International Standards for the Neurological Classification of Spinal Cord Injury, AIS American Spinal Injury Association Impairment Scale, CCI Charlston comorbidity index, VRPT volume restoration physiotherapy treatment

\section{Resource utilisation}

CIP diagnosis was associated with higher acute hospital resource utilisation in the first 2 weeks postinjury, with CIP cases requiring a higher number of radiological

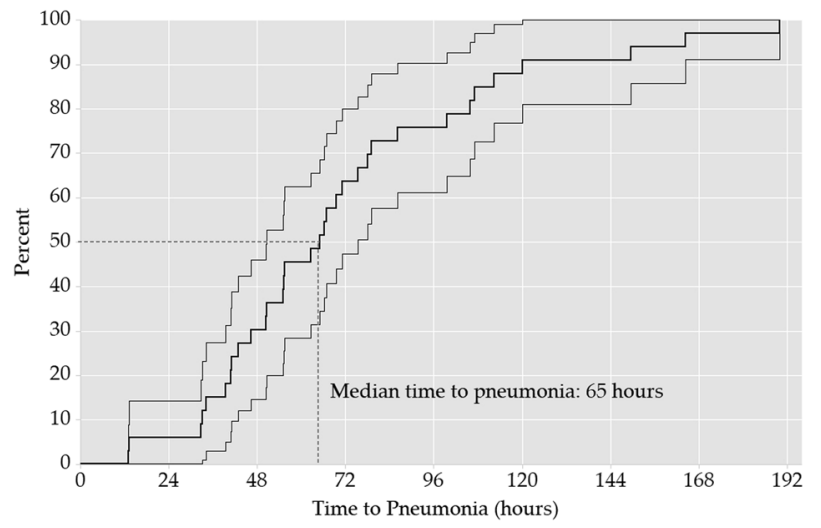

Fig. 1 Overall timing of clinically important pneumonia in spinal cord injury. Cumulative histogram showing the proportion of patients diagnosed with pneumonia at different times (dark grey line). The median time to CIP diagnosis is indicated by the dotted vertical light grey line, and $95 \%$ confidence intervals are indicated by the solid light grey line (colour figure online)

investigations, bronchoscopies and more frequent physiotherapy intervention. Additionally, CIP cases required an additional 6 days of recumbency, 8 days of ventilation and 7 days in the ICU, therefore prolonging their acute hospital LOS by 19 days, all of which were highly statistically significant (Table 3).

\section{Identification of CIP predictors}

Our multivariate analysis identified three baseline characteristics that were associated with the development of CIP. These were: being male, motor complete (AIS A or B) and having chest trauma (pneumothorax and/or haemothorax) on admission to hospital (Table 4). The overall $p$-value for the regression was $<0.001$. Interestingly, admission ISNCSCI level of injury did not influence pneumonia risk (OR: 0.24, 95\% CI, 0.04-1.42).

Five time-dependent covariates were then introduced into a Cox regression survival analysis modelling time to CIP diagnosis, where relative risk was described by a HR. A low number (5) of clinical interventions were considered, due to small sample size and small numbers of some levels of explanatory variables. Each clinical intervention was individually introduced and then removed from the model to ascertain its independent contribution to pneumonia risk. The survival analysis found that alongside the three baseline characteristics, time to intubation was the only timedependent covariate that significantly contributed to elevated CIP risk (HR 6.82, 95\% CI, 1.63-28.57, $p=0.009$ ).

As a high proportion $(n=38,51 \%)$ of individuals were intubated at the initiation of surgery, time to surgical initiation and time to intubation were introduced together into a multivariate Cox regression. The results of this analysis demonstrated that although the timing of surgery made 
Table 3 Resource utilisation in the acute hospital setting

\begin{tabular}{llll}
\hline & $\begin{array}{l}\text { CIP cases, } n=33 \\
\text { Median (IQR) }\end{array}$ & $\begin{array}{l}\text { No CIP cases, } n=60 \\
\text { Median (IQR) }\end{array}$ & $p$-Value \\
\hline Bronchoscopies (first 14 days) & $0(0-1)$ & $0(0-0)$ & $<0.0001$ \\
Chest X-rays (first 14 days) & $15(13-19)$ & $7.5(5-13)$ & $<0.0001$ \\
Time to first SOOB (days) & $13(9-18)$ & $7(5-10)$ & $<0.0001$ \\
Duration of ventilation (days) & $9.5(4.4-15.5)$ & $1.1(0-3.5)$ & $<0.0001$ \\
ICU LOS (days) & $11.5(8.2-16.9)$ & $4.8(2.7-7.9)$ & $<0.0001$ \\
Acute hospital LOS (days) & $42.8(24.8-51.9)$ & $23.9(18.1-39.2)$ & 0.002 \\
\hline
\end{tabular}

$C I P$ clinically important pneumonia, $I C U$ intensive care unit, $L O S$ length of stay, $S O O B$ sit out of bed

Table 4 All subsets regression

\begin{tabular}{llllll}
\hline Explanatory variable & Level & Baseline & Odds ratio & $95 \%$ CI & $p$-Value \\
\hline Gender & Male & Female & 18.29 & $1.91,174.9$ & 0.001 \\
Motor complete & Yes & No & 10.08 & $1.10,92.07$ & 0.011 \\
Chest trauma & Yes & No & 4.53 & $1.42,14.43$ & 0.007 \\
Intubation & $\mathrm{x}+1$ & $\mathrm{x}$ & $6.82^{\mathrm{a}}$ & $1.63,28.57$ & 0.009 \\
\hline
\end{tabular}

CI confidence interval

${ }^{\mathrm{a}}$ Hazard ratio was used

some contribution to the risk of CIP (HR 0.34, 95\% CI, $0.11-1.05, p=0.062)$, the timing of intubation had a larger relative risk (HR 8.17, 95\% CI, 1.91-34.92, $p=0.005$ ).

\section{Discussion}

This retrospective, multi-centre study identified four key predictors of CIP development in acute traumatic SCI; male gender, motor complete injury, presence of chest trauma and timing of intubation. Being male was the most important predictor identified in multivariate analysis (OR: 18.29, $p=0.001$ ), followed by motor complete $\mathrm{SCI}$ (OR: $10.08, p$ $=0.011$ ) and presence of chest trauma (OR: 4.53, $p=0.007)$. Additionally, every day of delay to intubation was associated with increased risk (HR: 8.17, $p=0.05$ ), which appeared to be independent of surgical timing.

\section{Important baseline predictors}

Being male was associated with 18 times the risk of CIP diagnosis. Although this is the first study to identify gender as being a key predictor of pneumonia in a traumatic SCI cohort, this result does align with previous research in the trauma population [15]. Contributing factors may include the poorer general health and higher alcohol consumption of males. Although we did not find comorbidity burden to be a significant contributor to pneumonia risk, however, the baseline clinical characteristics of our sample demonstrate that males were $9 \%$ more likely to be intoxicated at time of injury. This is consistent with the traumatic SCI literature, where intoxication at the scene has been demonstrated to elevate pneumonia risk [10].

Motor complete injuries (AIS A or B) were associated with 10 times the risk of CIP compared with motor incomplete (AIS C or D) injuries, whereas ISNCSCI level of injury was not considered significantly predictive. Motor complete injuries result in flaccid paralysis of the intercostal and abdominal muscles below the level of injury, destabilising the chest wall and resulting in an inefficient, paradoxical respiratory pattern. This mechanical disadvantage is particularly exaggerated in cervical injuries and culminates in lower tidal volumes with increased ventilation of pulmonary dead space, hypersecretion, weak cough, fatigue and a tendency towards distal airway collapse and microatelectasis. Our findings are consistent with the literature that severity of spinal cord damage increases in-hospital morbidity and specifically pneumonia risk [9]. Perhaps uniquely and importantly in thoracolumbar SCI, the combination of chest trauma and motor complete injury is key and explains worsened respiratory function in the setting of known greater chest wall innervation in this subset of our cohort.

The presence of chest trauma (pneumothorax and/or haemothorax) equated to five times the risk of CIP, physiologically explained by passive atelectasis caused by loss of contact between parietal and visceral pleura. The presence of chest trauma limits the safety and efficacy of volume restoration techniques commonly utilised by physiotherapists to treat atelectasis such as ventilator or manual hyperinflation, in/exsufflation and intermittent positive pressure breathing. A lower or gentler dose of VRPT may predispose these cases to occurrence or progression of 
infection. SCI individuals with chest trauma may also be at a greater risk of CIP due to pulmonary contusions, which commonly occur following blunt chest injury. Pulmonary contusions result in sputum plugging with thick haematitic secretions, bronchospasm and hypersecretion, causing reduced pulmonary compliance and functional residual capacity, the effects of which peak $72 \mathrm{~h}$ after injury. Although there have been no studies to date isolating chest trauma as a predictor of pneumonia in the SCI population, our result aligns with the able-bodied trauma population, where haemothoraces, pulmonary contusions and multiple rib fractures have been demonstrated to be key predictors [16].

\section{Important clinical predictors}

The timing of intubation was the primary clinical intervention found to contribute to pneumonia risk, with pneumonia hazard increasing eightfold with every additional day of delay to intubation. This result is clinically important, as presumably individuals with severe respiratory insufficiency are also likely to develop CIP. If high-risk individuals are intubated earlier, our results indicate that this reduces their risk of CIP development. To our knowledge, no studies have included the timing of intubation in their analysis of pneumonia predictors in the SCI population. However, there is some evidence that early elective intubation is beneficial in the moderately injured trauma population, particularly in cases presenting with chest trauma. In these cases, early elective intubation has been demonstrated to lower mortality (from $12 \%$ to $2 \%$ ) [17] and morbidity (reducing pneumonia risk and ICU LOS) [18]. The benefits of early elective intubation in the SCI population may outweigh the risks, enabling adequate tidal volumes, lung recruitment and secretion clearance. Interestingly, the predominant reason for intubation in our study was initiation of surgery ( $n=39,53 \%)$, however, the timing of surgery was not found to be significantly predictive despite the strength of the literature in this area. Considering the median time to spinal surgery was within $24 \mathrm{~h}$ of injury in this study, it may be that other clinical factors become of greater importance once surgery is performed within this early timeframe.

\section{Incidence and timing of pneumonia}

The incidence of CIP in this study was $35 \%(n=33)$. There are no universally accepted, gold standard diagnostic criteria for pneumonia and clinical practice guidelines lack specificity [19]. The variability in incidence reported in the literature may simply reflect the variability in criteria for CIP. For this reason in this study, we defined the criteria for pneumonia and its severity.
The median time to CIP diagnosis in this study was $65 \mathrm{~h}$ (IQR: 42-93). HAP occurred following $\geq 48$ h of ventilation in most $(78 \%)$ cases, which is somewhat paradoxical given that earlier intubation was shown to reduce CIP risk. Intubation reduces respiratory insufficiency, which is at its worst in the first $72 \mathrm{~h}$ of injury by providing mechanical support and avoiding hypoventilation. We have demonstrated that early intubation significantly reduces CIP risk and therefore the timing of intubation may be critical to minimise respiratory complications and of greater importance than duration of ventilation.

Interestingly a significant proportion of CIP cases $(n=$ $10,30 \%$ ) occurred within $48 \mathrm{~h}$ of hospital admission. Acute aspiration may be responsible for this early incidence. Additionally, a higher proportion of patients with CIP were initially admitted to a pre-surgical hospital, which has been shown in the literature to impact complication rates [20]. Overall, of the cases requiring invasive ventilation, most ( $n$ $=72,97 \%$ ) were self-ventilating until after acute hospital admission. Intubating SCI individuals at the scene, particularly when a cervical spine fracture is suspected, can be risky and is generally utilised only in the setting of severe cardiorespiratory instability or altered conscious state. In the absence of an endotracheal tube, SCI individuals are at high risk of aspiration due to positioning with spinal immobilisation, high analgesia requirements and deficits in airway protection (reduced FVC, weak cough).

\section{Clinical impact of pneumonia}

Cases with CIP were ventilated for a median of 8 days longer, stayed in ICU for an additional 7 days and required inpatient acute care for an extra 19 days (Table 3), all of which were highly statistically significant. These results underscore the burden that moderate and severe respiratory tract infections have on the healthcare system.

\section{Study strengths and limitations}

This study reports the current incidence and clinical impact of CIP in a cohort of critically ill traumatic SCI individuals and identifies four independent predictors that elevate CIP risk. Data were manually collected from the medical record by a senior physiotherapist, which facilitated data completeness, and CIP was well defined.

Although our findings were significant, our study has methodological limitations that impact its clinical utility, reproducibility and external validity. These include its retrospective nature, small sample size, inclusion and exclusion criteria and lack of a validation cohort. Of note are the baseline differences between patients excluded due to key information unavailable and our included sample, which introduces selection bias. Additionally, patients with a 
purely radiological definition of TCCS and those with ISNCSCI C1-2 injury levels were excluded, which may impact the clinical extrapolation of our results in an allencompassing SCI cohort. Furthermore, our sample was taken from a single high-income country and the proportion of cases resulting from different injury mechanisms (e.g., penetrating trauma) may vary between regions. The definition of CIP used in this study has not been previously published or validated, and due to large numbers of excluded independent variables there is potential for inaccuracy in our results.

In conclusion, baseline characteristics such as male gender, motor complete injury, presence of chest trauma and the timing of intubation are key early predictors involved in the onset of CIP in critically ill acute traumatic SCI individuals. The median time to spinal surgery was $22 \mathrm{~h}$ in this study and therefore ideal to minimise pneumonia risk, possibly unmasking other previously unidentified predictors. This study enables the potential implementation of targeted, proactive interventions in high-risk individuals to reduce severity or presence of infection, thereby reducing in-hospital morbidity and overall hospital costs.

Acknowledgements Many thanks to Jim Sayer who assisted with data organisation and Sue Finch who assisted with statistical analysis.

Funding This study is supported by the National Health and Medical Research Council; and by the Transport Accident Commission, through the Institute for Safety, Compensation and Recovery Research.

Author contributions JA was responsible for the literature search, study design, data collection, data analysis, data interpretation, referencing, writing and critical revision. PEB was responsible for the study design, data analysis, data interpretation, writing and critical revision. CRB was responsible for the study design, writing and critical revision.

\section{Compliance with ethical standards}

Conflict of interest The authors declare that they have no conflict of interest.

\section{References}

1. Burns SP. Acute respiratory infections in persons with spinal cord injury. Phys Med Rehabil Clin N Am. 2007;18:203-16.

2. Ball PA. Critical care of spinal cord injury. Spine. 2001;26: S27-S30.

3. Berney S, Bragge P, Granger C, Opdam H, Denehy L. The acute respiratory management of cervical spinal cord injury in the first 6 weeks after injury: a systematic review. Spinal Cord. 2011;49:17-29.
4. Jackson AB, Groomes TE. Incidence of respiratory complications following spinal cord injury. Arch Phys Med Rehabil. 1994;75:270-5.

5. van Weert KCM, Schouten EJ, Hofstede J, van de Meent H, Holtslag HR, van den Berg-Emons RJG. Acute phase complications following traumatic spinal cord injury in Dutch level 1 trauma centres. J Rehabil Med. 2014;46:882-5.

6. Winslow C, Bode RK, Felton D, Chen D, Meyer PR. Impact of respiratory complications on length of stay and hospital costs in acute cervical spinal cord injury. Chest. 2002;121:1548-54.

7. Walter EJ, Carraretto M. The neurological and cognitive consequences of hyperthermia. Crit Care. 2016;20:199-206.

8. Bourassa-Moreau E, Mac-Thiong JM, Feldman DE, Thompson C, Parent S. Non-neurological outcomes after complete traumatic spinal cord injury: the impact of surgical timing. J Neurotrauma. 2013;30:1596-601.

9. Wilson JR, Arnold PM, Singh A, Kalsi-Ryan S, Fehlings MG. Clinical prediction model for acute inpatient complications after traumatic cervical spinal cord injury: a subanalysis from the Surgical Timing in Acute Spinal Cord Injury Study. J Neurosurg Spine. 2012;17:46-51.

10. CLn Crutcher, Ugiliweneza, Hodes B, Kong JE, Boakye M. Alcohol intoxication and its effects on traumatic spinal cord injury outcomes. J Neurotrauma. 2014;31:798-802.

11. Agostinello J, Battistuzzo CR, Skeers P, Bernard S, Batchelor PE. Early spinal surgery following thoracolumbar spinal cord injury: process of care from trauma to theater. Spine. 2017;42:E617-23.

12. Battistuzzo CR, Armstrong A, Clark J, Worley L, Sharwood L, Lin $\mathrm{P}$, et al. Early decompression following cervical spinal cord injury: examining the process of care from accident scene to surgery. J Neurotrauma. 2016;33:1161-1169.

13. Garner JS, Jarvis W, Emori TG, Horan T, Hughes JM. CDC definitions for nosocomial infections, 1988. Am J Infect Control. 1988;16:128-40.

14. Burnham KP, Anderson DR. Model selection and multimodel inference: a practical information-theoretical approach. 2nd edn. New York: SpringerVerlag; 2002.

15. Gannon CJ, Pasquale M, Tracy JK, McCarter RJ, Napolitano LM. Male gender is associated with increased risk for postinjury pneumonia. Shock. 2004;21:410-4.

16. Michelet P, Couret D, Bregeon F, Perrin G, D'Journo XB, Pequignot $\mathrm{V}$, et al. Early onset pneumonia in severe chest trauma: a risk factor analysis. J Trauma Inj Infect Crit Care. 2010;68:395-400.

17. Miraflor E, Chuang K, Miranda MA, Dryden W, Yeung L, Strumwasser A, et al. Timing is everything: delayed intubation is associated with increased mortality in initially stable trauma patients. J Surg Res. 2011;170:286-90.

18. Huf R, Kraft S, Schildberg FW. Effect of intubation timing on the clinical course of polytrauma patients. Zent Chir. 1996;121:21-3.

19. Kalil AC, Metersky ML, Klompas M, Muscedere J, Sweeney DA, Palmer LB, et al. Management of adults with hospital-acquired and ventilator-associated pneumonia: 2016 clinical practice guidelines by the Infectious Diseases Society of America and the American Thoracic Society. Clin Infect Dis. 2016;63:e61-e111.

20. Richard-Denis A, Feldman D, Thompson C, Mac-Thiong J. Prediction of functional recovery six months following traumatic spinal cord injury during acute care hospitalization. J Spinal Cord Med. 2017;15:1-9. 